\section{Original Article}

Infectious Diseases, Microbiology \& Parasitology

\section{Check for updates}

\section{OPEN ACCESS}

Received: Jun 22, 2018

Accepted: Oct 23, 2018

Address for Correspondence:

Eun Hwa Choi, MD, FIDSA

Department of Pediatrics, Seoul National

University Children's Hospital, 101 Daehak-ro,

Jongno-gu, Seoul 03080, Korea.

E-mail: eunchoi@snu.ac.kr

c) 2019 The Korean Academy of Medical

Sciences.

This is an Open Access article distributed under the terms of the Creative Commons Attribution Non-Commercial License (https:// creativecommons.org/licenses/by-nc/4.0/) which permits unrestricted non-commercial use, distribution, and reproduction in any medium, provided the original work is properly cited.

ORCID IDs

Youn Young Choi (iD)

https://orcid.org/0000-0001-8749-0951 Mi Seon Han (D)

https://orcid.org/0000-0002-3896-1400

Hoan Jong Lee (iD

https://orcid.org/0000-0001-9643-3692 Ki Wook Yun (D)

https://orcid.org/0000-0002-0798-6779 Chang Ho Shin (D)

https://orcid.org/0000-0002-4154-7964 Won Joon Yoo (iD)

https://orcid.org/0000-0001-5225-1440

Tae-Joon Cho (D)

https://orcid.org/0000-0001-8514-377X

\title{
Mycobacterium bovis Osteitis Following Immunization with Bacille Calmette-Guérin (BCG) in Korea
}

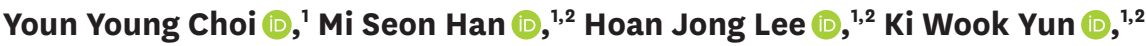

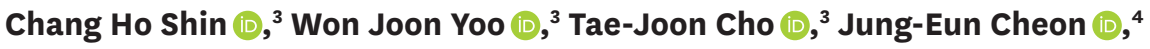 \\ Kyoung Un Park (iD, ${ }^{5}$ and Eun Hwa Choi ${ }^{10}{ }^{1,2}$ \\ 'Department of Pediatrics, Seoul National University Children's Hospital, Seoul, Korea \\ ${ }^{2}$ Department of Pediatrics, Seoul National University College of Medicine, Seoul, Korea \\ ${ }^{3}$ Division of Pediatric Orthopaedics, Seoul National University Children's Hospital, Seoul, Korea \\ ${ }^{4}$ Department of Radiology, Seoul National University Children's Hospital, Seoul, Korea \\ ${ }^{5}$ Department of Laboratory Medicine, Seoul National University Bundang Hospital, Seongnam, Korea
}

\section{ABSTRACT}

Background: Mycobacterium bovis Bacille Calmette-Guérin (BCG) osteitis, a rare complication of BCG vaccination, has not been well investigated in Korea. This study aimed to evaluate the clinical characteristics of BCG osteitis during the recent 10 years in Korea.

Methods: Children diagnosed with BCG osteitis at the Seoul National University Children's Hospital from January 2007 to March 2018 were included. M. bovis BCG was confirmed by multiplex polymerase chain reaction (PCR) in the affected bone. BCG immunization status and clinical information were reviewed retrospectively.

Results: Twenty-one patients were diagnosed with BCG osteitis and their median symptom onset from BCG vaccination was 13.8 months (range, 6.0-32.5). Sixteen children $(76.2 \%)$ received Tokyo-172 vaccine by percutaneous multiple puncture method, while four $(19.0 \%)$ and one $(4.8 \%)$ received intradermal Tokyo-172 and Danish strain, respectively. Common presenting symptoms were swelling $(76.2 \%)$, limited movement of the affected site $(63.2 \%)$, and pain $(61.9 \%)$ while fever was only accompanied in $19.0 \%$. Femur $(33.3 \%)$ and the tarsal bones $(23.8 \%)$ were the most frequently involved sites; and demarcated osteolytic lesions (63.1\%) and cortical breakages (42.1\%) were observed on plain radiographs. Surgical drainage was performed in $90.5 \%$, and $33.3 \%$ of them required repeated surgical interventions due to persistent symptoms. Antituberculosis medications were administered for a median duration of 12 months (range, 12-31). Most patients recovered without evident sequelae.

Conclusion: Highly suspecting BCG osteitis based on clinical manifestations is important for prompt management. A comprehensive national surveillance system is needed to understand the exact incidence of serious adverse reactions following BCG vaccination and establish safe vaccination policy in Korea.

Keywords: Mycobacterium bovis; BCG; Osteitis; Vaccination; Complications 
Jung-Eun Cheon (iD)

https://orcid.org/0000-0003-1479-2064

Kyoung Un Park iD

https://orcid.org/0000-0002-2402-7633

Eun Hwa Choi iD

https://orcid.org/0000-0002-5857-0749

\section{Presentation}

This study was presented in part at the 2018

American Society for Microbiology Microbes for Clinical Infections and Vaccines in Atlanta, Georgia, Unites States, June 7-11, 2018.

Disclosure

The authors have no potential conflicts of interest to disclose.

\section{Author Contributions}

Conceptualization: Yun KW, Shin $\mathrm{CH}$, Yoo WJ, Cho TJ, Cheon JE, Park KU. Data curation: Yun KW, Shin $\mathrm{CH}$, Yoo WJ, Cho TJ, Cheon JE, Park KU. Formal analysis: Yun KW, Shin $\mathrm{CH}$, Yoo WJ, Cho TJ, Cheon JE, Park KU. Investigation: Choi YY, Han MS. Supervision: Lee HJ, Choi EH. Writing - original draft: Choi YY, Han MS. Writing - review \& editing: Choi EH, Lee HJ.

\section{INTRODUCTION}

Bacille Calmette-Guérin (BCG) immunization is recommended to prevent disseminated tuberculosis and extrapulmonary tuberculosis including meningitis in young children. BCG is a live attenuated vaccine developed from serial passage of Mycobacterium bovis. Currently the Russian (Moscow-368), the Bulgarian (Sofia SL222) and the Tokyo-172 are the three most commonly used BCG strains worldwide. ${ }^{1}$ The World Health Organization recommends intradermal administration of the vaccine, but percutaneous application using a multiple puncture injection device is practiced in some countries including Japan and Korea. The Tokyo-172 BCG vaccine is currently available in both percutaneous and intradermal formulations.

Although adverse events at injection site are frequently observed, serious adverse events following BCG vaccination are rare. The incidence of dissemination of BCG is estimated to be $0.19-1.56$ per million vaccinations. ${ }^{2}$ BCG osteitis is another rare systemic adverse reaction following BCG vaccination. The cases of BCG osteitis were increasingly notified when there was a change in the vaccine strain, for example, in Scandinavia and in Eastern Europe. ${ }^{2}$ More recently, there are increased reports of osteitis in Japan, Taiwan, and Thailand. ${ }^{3-5}$

In Korea, Japan and Taiwan, BCG vaccination is recommended for all infants through a national immunization program (NIP). Infants in Japan receive the BCG Tokyo (strain 172; Japan BCG Laboratory) by percutaneous method, whereas those in Taiwan receive the BCG Tokyo (strain 172; Taiwan CDC Vaccine Center) by intradermal injection. In Korea, newborns are vaccinated with either intradermal BCG Danish (strain 1331; Statens Serum Institute), intradermal BCG Tokyo (strain 172; Japan BCG Laboratory), or percutaneous BCG Tokyo (strain 172; Japan BCG Laboratory). Previously, the incidence of osteitis following BCG Tokyo-172 vaccination was reported to be extremely low, hence the BCG Tokyo vaccine was considered as the safest BCG vaccine. ${ }^{6,7}$ However, a higher incidence of 3.68 cases per million vaccinations was reported in Taiwan. ${ }^{8}$

Unlike Japan and Taiwan, no long-term observational studies of serious adverse reactions following BCG vaccinations have been reported in Korea. Here, we describe the clinical characteristics of BCG osteitis from our experience during the recent 10 years.

\section{METHODS}

\section{Study subjects}

A retrospective chart review was performed on all laboratory-confirmed BCG osteitis children admitted to Seoul National University Children's Hospital, a large tertiary referral center in Korea, from January 2007 to March 2018. Children who were clinically suspected of BCG infection but not confirmed by BCG-specific polymerase chain reaction (PCR) were excluded. History of BCG vaccination was taken from the data in the national immunization registry, namely, the brand name of BCG vaccine, manufacturer, and the date and the site of vaccination. For those whose data are not available from the registry, BCG vaccination history was interpreted from the personal immunization records and inspecting the scar of the injection sites. Clinical data were collected regarding patient demographics, presenting symptoms, physical findings, radiologic features, histologic findings, and clinical outcome. The immune status was evaluated by the number and percentage of T-cell subsets, complement levels, immunoglobulin levels, and performing the dihydrorhodamine test. 


\section{Laboratory confirmation}

The presumptive diagnosis in the majority of the cases was mainly based on positive PCR for Mycobacterium tuberculosis complex directly from the isolates of M. bovis BCG, or from the fresh samples obtained from the affected tissue. Laboratory confirmation of $M$. bovis BCG was performed with the use of the same protocol previously described by Kim et al. 9 Briefly, the real-time PCR targeted for the 53-bp mycobacterial interspersed repetitive units (MIRUs) of the senX3-regX3 IR was performed to differentiate $M$. bovis BCG from non-BCG M. tuberculosis complex. Subsequently, a multiplex PCR assay with 7 primers (ET1, ET2, ET3, RD81, RD8r, RD14l, and RD14r) that amplified 3 regions of difference - RD1, RD8 and RD14 was performed to discriminate among the BCG substrains.

\section{Statistical analysis}

Categorical variables were summarized by absolute frequencies and percentages. Continuous variables were expressed as means with standard deviation or - if skewed - as medians with ranges. All data management and analysis were performed using SPSS version 19.0 (IBM Corp., Armonk, NY, USA).

\section{Ethics statement}

This study was approved by the Institutional Review Boards (IRB) of Seoul National University Hospital (IRB No. H-1711-120-901). Informed consent of the patients was waived.

\section{RESULTS}

\section{Patient characteristics}

During the study period, 21 BCG osteitis cases were confirmed. Three children who are highly suspicious of having BCG osteitis from the histologic findings were excluded because BCGspecific PCR was not performed. Detailed clinical characteristics of the patients are shown in Table 1. Of these, 4 cases had been previously reported. ${ }^{9,10}$ Fourteen patients $(66.7 \%)$ were boys and seven were girls. The symptoms or signs of osteitis were first noticed at the median age of 14.3 months (range, 6.5-33.4) (Table 2). The median age at BCG inoculation was 24 days (range, 2-36). Sixteen patients $(76.2 \%)$ received BCG Tokyo-172 strain by percutaneous multiple puncture method, four (19.0\%) received BCG Tokyo-172 strain intradermally, and one (4.8\%) received Danish BCG intradermally. A total of 20 cases $(95.2 \%)$ were inoculated with Tokyo-172 strain. The years the BCG osteitis patients received vaccination are shown in Fig. 1. Patients were vaccinated in different regions: Seoul-metropolitan (35\%), central area $(40 \%)$, and southern area $(25 \%)$.

\section{Clinical manifestations}

The median duration from BCG vaccination to symptom onset was 13.8 months (range, 6.0-32.5). Patients were diagnosed after a median of 29 days (range, 3-112) from symptom onset. At the time of diagnosis, the most common symptoms were swelling $(76.2 \%)$, limitation of movement $(63.2 \%)$ and pain $(61.9 \%)$. Fever $\left(\geq 38.0^{\circ} \mathrm{C}\right)$ was accompanied only in $19.0 \%$. Excepting the two cases with multiple bone involvement, the most commonly affected site was femur (33.3\%), followed by the tarsal bones (28.6\%), tibia (9.5\%), and rib and sternum (9.5\%), as shown in Table 2. The initial diagnosis for the 19 referred cases were bacterial osteomyelitis in $10(52.6 \%)$, bone tumor or Langerhans cell histiocytosis in 6 (31.6\%), and transient synovitis in $3(15.7 \%)$. 
Table 1. Clinical characteristics of the 21 patients with BCG osteitis

\begin{tabular}{|c|c|c|c|c|c|c|c|c|c|c|c|}
\hline No. & $\begin{array}{c}\text { Route } \\
\text { of } \\
\text { BCG }\end{array}$ & $\begin{array}{l}\text { BCG } \\
\text { strain }\end{array}$ & $\begin{array}{c}\text { Age at } \\
\text { vaccination, } \\
\text { day }\end{array}$ & $\begin{array}{l}\text { Age at } \\
\text { onset, } \\
\text { mon }\end{array}$ & $\begin{array}{l}\text { Duration from } \\
\text { vaccination to } \\
\text { onset, mon }\end{array}$ & Clinical manifestation & $\begin{array}{l}\text { Pathologic } \\
\text { finding }\end{array}$ & $\begin{array}{l}\text { AFB stain/ } \\
\text { culture }\end{array}$ & $\begin{array}{l}\text { Tissue } \\
\text { MTB PCR }\end{array}$ & $\begin{array}{l}\text { Medication/total } \\
\text { duration, mon }\end{array}$ & $\begin{array}{l}\text { Clinical } \\
\text { outcome }\end{array}$ \\
\hline 1 & PC & Tokyo & 30 & 11 & 9 & Right knee pain for 2 wk & $\mathrm{CG}+\mathrm{CN}$ & $-/+$ & Not done & HRSZ-HRZ-HR/16 & Good \\
\hline 2 & PC & Tokyo & 36 & 14 & 12 & Left ankle swelling for 3 wk & $\mathrm{CG}+\mathrm{CN}$ & $-1+$ & - & HRSZ-HRZ-HR/14 & $\begin{array}{l}\text { Length } \\
\text { discrepancy }\end{array}$ \\
\hline 3 & PC & Tokyo & 25 & 20 & 19 & Left knee pain for 3 day & $\mathrm{CG}+\mathrm{CN}$ & $-1-$ & + & $\begin{array}{l}\text { HRSZ-HRZ- } \\
\mathrm{HRZ}+\mathrm{CLA} / 31^{\mathrm{a}}\end{array}$ & Good \\
\hline 4 & PC & Tokyo & 23 & 24 & 23 & Left leg pain for 3 mon & $\mathrm{CG}+\mathrm{CN}$ & $+1-$ & + & $\mathrm{HR} / 12$ & Good \\
\hline 5 & PC & Tokyo & 2 & 21 & 20 & Left ankle pain for 1 mon & $\mathrm{CG}+\mathrm{CN}$ & $-/+$ & + & HRZ-HR/12 & Good \\
\hline 6 & PC & Tokyo & Unknown & 15 & Unknown & Left leg limping for 3 mon & $\mathrm{CG}+\mathrm{CN}$ & $+/+$ & + & HRZ-HR/12 & Good \\
\hline 7 & PC & Tokyo & 18 & 18 & 17 & $\begin{array}{l}\text { Right knee painful swelling } \\
\text { for } 4 \text { day }\end{array}$ & Nonspecific & $-1-$ & Not done & $\mathrm{HR} / 12$ & Good \\
\hline 8 & PC & Tokyo & 30 & 16 & 14 & Left leg limping for 1 mon & $\mathrm{CG}+\mathrm{CN}$ & $-/+$ & Not done & HRZ-HR/12 & Good \\
\hline 9 & PC & Tokyo & 29 & 34 & 32 & $\begin{array}{l}\text { Left elbow swelling and pain } \\
\text { for } 2 \text { day }\end{array}$ & Not done & $+/+$ & + & HREZ-HR/12 & Good $^{b}$ \\
\hline 10 & PC & Tokyo & 15 & 11 & 10 & Forehead swelling for 3 mon & $\mathrm{CG}+\mathrm{CN}$ & $-1+$ & + & $\mathrm{HRZ}-\mathrm{HR} / 12$ & Good \\
\hline 11 & PC & Tokyo & 17 & 16 & 14 & Left leg limping for 3 mon & $C G+C N$ & $-1+$ & Not done & HREZ-HR/12 & Good \\
\hline 12 & PC & Tokyo & 24 & 17 & 16 & Right leg limping for 3 day & CG & $-1+$ & + & HREZ-HR/12 & Good \\
\hline 13 & PC & Tokyo & 21 & 7 & 6 & Right leg swelling for 1 mon & Not done & $-/+$ & + & HRZ-HR/12 & Good \\
\hline 14 & PC & Tokyo & 11 & 15 & 14 & $\begin{array}{l}\text { Right leg pain and limping } \\
\text { for } 1 \text { mon }\end{array}$ & $\mathrm{CG}+\mathrm{CN}$ & $+/+$ & + & HRZ-HR/12 & Good \\
\hline 15 & ID & Danish & 24 & 7 & 6 & Left ankle swelling for 3 wk & Not done & $-1-$ & + & HRZ-HR/12 & Good \\
\hline 16 & $\mathrm{PC}$ & Tokyo & 15 & 19 & 18 & $\begin{array}{l}\text { Left knee swelling and } \\
\text { limping gait for } 2 \mathrm{wk}\end{array}$ & $\begin{array}{c}\text { Chronic } \\
\text { inflammation }\end{array}$ & $-1-$ & + & $\mathrm{HR} / 12$ & Good \\
\hline 17 & PC & Tokyo & 26 & 10 & 8 & Right wrist mass for 2 mon & CG & $+/+$ & + & HRZ-HR/12 & Good \\
\hline 18 & ID & Tokyo & 16 & 7 & 5 & Left foot swelling for 1 mon & $\mathrm{CG}+\mathrm{CN}$ & $-1+$ & + & HRZ-HR/12 & Good \\
\hline 19 & ID & Tokyo & 33 & 10 & 9 & Chest wall swelling for 1 mon & $\mathrm{CG}+\mathrm{CN}$ & $-1-$ & + & $\mathrm{HR} / 12$ & Good \\
\hline 20 & ID & Tokyo & 32 & 14 & 13 & Left rib mass for 2 wk & $\mathrm{CG}+\mathrm{CN}$ & $-1+$ & + & HRZ-HR/12 & Good \\
\hline 21 & ID & Tokyo & 27 & 10 & 8 & Right ankle swelling for 2 wk & $\mathrm{CG}+\mathrm{CN}$ & $\begin{array}{l}\text { Not done/ } \\
\text { Not done }\end{array}$ & Not done & $\mathrm{HR} / 12$ & Good \\
\hline
\end{tabular}

$\mathrm{BCG}=$ Bacille Calmette-Guérin, $\mathrm{AFB}=$ acid fast bacilli, $\mathrm{MTB} \mathrm{PCR}=\mathrm{M}$. tuberculosis complex polymerase chain reaction, $\mathrm{PC}=$ percutaneous, CG = chronic granuloma, $\mathrm{CN}=$ caseous necrosis, $\mathrm{H}=$ isoniazid, $\mathrm{R}=$ rifampin, $\mathrm{S}=$ streptomycin, $\mathrm{Z}=$ pyrazinamide, $\mathrm{CLA}=$ clarithromycin, $\mathrm{E}=$ ethambutol, $\mathrm{ID}=$ intradermal. ${ }^{a}$ This patient received prolonged treatment with various antimycobacterial combinations as making a definitive diagnosis was difficult; ${ }^{\mathrm{b}}$ This patient recovered without joint complication until the patient developed osteomyelitis at the same joint caused by Mycobacterium intracellulare.

\section{Comorbidities}

Three (14.3\%) were born preterm at 32-36 weeks of gestational age with a range of birth weight from $2.4 \mathrm{~kg}$ to $2.8 \mathrm{~kg}$. Two had a previous history of lymph node excision due to BCG lymphadenopathy. Tests for human immunodeficiency virus were performed in 18 patients and all were negative. Further immunological investigations performed in 15 cases were all normal at the time of diagnosis. Although there were no identified cases of primary immunodeficiency, one had a past history of Pneumocystis jirovecii pneumonitis during infancy and thus was suspected of immunodeficiency.

\section{Laboratory and radiologic findings}

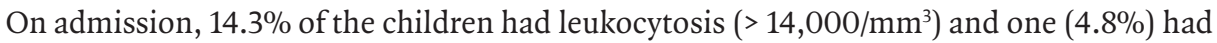
leukopenia $\left(<4,000 / \mathrm{mm}^{3}\right)$. C-reactive protein was elevated $(>5.0 \mathrm{mg} / \mathrm{L})$ in $35 \%$. Erythrocyte sedimentation rate was tested for 16 cases and was elevated (20 mm/hr or greater) in $68.8 \%$. Solitary demarcated osteolytic lesion (63.1\%) and cortical breakage (42.1\%) were the most common findings on initial plain radiographs in 19 cases. In four patients $(21.0 \%)$ there were only soft tissue swelling or bulging on plain radiographs. Magnetic resonance imaging was performed in 20 patients including those with no evident bony abnormality on simple radiographs. Bone abnormalities were found on magnetic resonance imaging in all cases. The epiphysis was affected in $6(50 \%)$ of the 12 patients with BCG osteitis in the long bones. Transphyseal spread between the epiphysis and the metaphysis was observed in $3(30 \%)$. 
Table 2. Overall clinical characteristics of BCG osteitis cases from January 2007 to March 2018

\begin{tabular}{lc}
\hline Characteristics & No. of cases (\%) \\
\hline Gender & $14(66.7)$ \\
Men & $7(33.3)$ \\
Women & $14.3(6.5-33.4)$ \\
Median age at onset, mon (range) & $24.0(2-36)$ \\
BCG vaccine & \\
Danish-1331, intradermal & $1(4.8)$ \\
Tokyo-172, intradermal & $4(19.0)$ \\
Tokyo-172, percutaneous & $16(76.2)$ \\
Symptoms and signs & \\
Swelling & $16(76.2)$ \\
Limited movement & $12(63.2)$ \\
Pain & $13(61.9)$ \\
Fever & $4(19)$ \\
Involved sites & \\
Femur & $7(33.3)$ \\
Tarsal bones & $6(28.6)$ \\
Tibia & $2(9.5)$ \\
Sternum and rib & $2(9.5)$ \\
Humerus & $1(4.8)$ \\
Radius & $1(4.8)$ \\
Phalanx & $1(4.8)$ \\
Multiple & $2(9.5)$ \\
\hline BCG &
\end{tabular}

$\mathrm{BCG}=$ Bacille Calmette-Guérin.

Danish-intradermal Tokyo-intradermal Tokyo-percutaneous

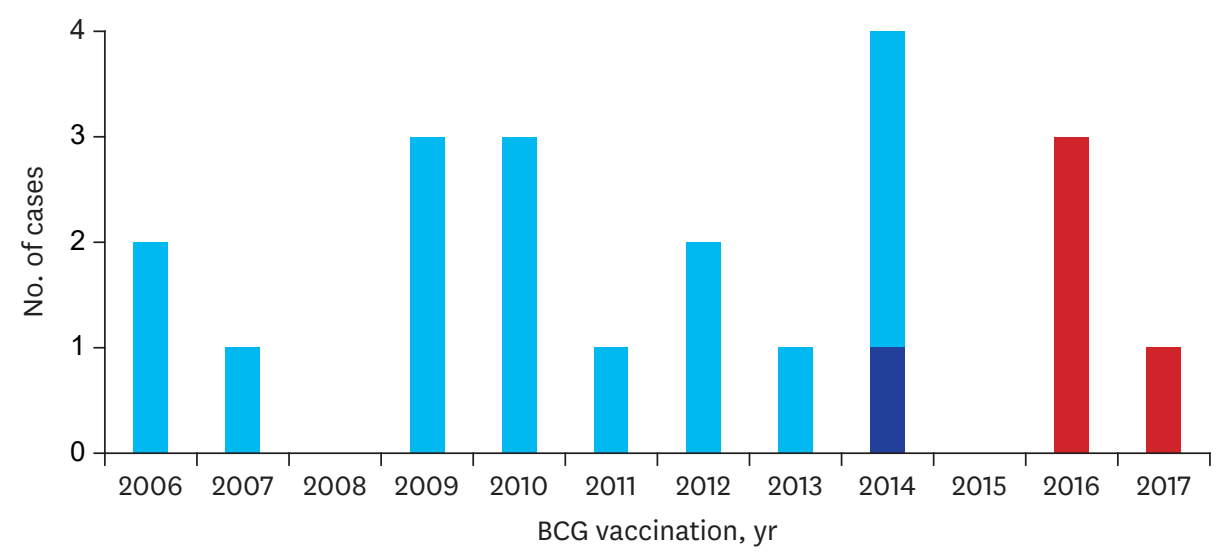

Fig. 1. Number of BCG osteitis patients vaccinated in each year. $B C G=$ Bacille Calmette-Guérin .

No diaphyseal involvement was noticed. Soft tissue abnormalities were common, where the most common finding was inflammation of the surrounding muscles $(87.5 \%)$, followed by soft tissue abscess (43.8\%). Subcutaneous inflammation was noted in 37.5\%. Eight of the 16 cases with a single bone lesion had joint involvement, including four cases with synovial hypertrophy and four cases with joint effusion.

\section{Microbiological findings}

The diagnosis of $M$. bovis BCG osteitis was confirmed in all patients by performing BCGspecific PCR on DNA extracted from biopsy samples or bacterial isolates. The real-time PCR for the 53-bp MIRU was all negative, suggesting M. bovis BCG strains. The multiplex PCR assay with 7 primers that can discriminate BCG substrains revealed the Tokyo strain in 20 and 
Danish strain in one. Acid-fast bacilli stain was positive in $25.0 \%$ and $M$. bovis grew by culture in $70.0 \%$. PCR for $M$. tuberculosis complex on fresh biopsy sample was positive in $93.8 \%$. Histopathology showed chronic granuloma with caseous necrosis in $77.8 \%$.

\section{Treatment}

In our case series, $20(95.2 \%)$ children underwent surgical procedures, where 19 patients had surgical treatment and one received biopsy only. Seven (33.3\%) patients required more than one surgical interventions because of persistent swelling and spread of infection outside the epiphysis. All patients received antimycobacterial medication with isoniazid and rifampicin for a median duration of 12 months (range, 12-31).

\section{Clinical outcome}

Eighteen of the 21 patients were followed-up for more than one year and three children were still on treatment. The median follow-up duration was 3.15 years (range, 1.0-10.8). All were compliant with their medical treatment and were regularly followed-up every 1 to 2 months. Symptoms, physical examination findings and radiographic findings improved in most patients $(95.2 \%)$ without obvious sequelae. There was no recurrence of BCG osteitis after completing antimycobacterial therapy. One patient who had a talar lesion with inflammatory destruction and necrotic fragmentation at the time of diagnosis later developed progressive bony remodeling with collapse of the talus, resulting in leg length shortening. Another patient developed joint contracture due to nontuberculosis mycobacterial infection in the previous BCG osteitis site at the age of 5 years. This patient, who had a past history of P. jirovecii pneumonitis during infancy, suffered from disseminated nontuberculous mycobacterial infection. One patient had horseshoe kidney, tethered cord syndrome and developmental delay at the time of BCG osteitis, and was diagnosed with Kabuki syndrome with KMT2D (also known as MLL2) gene mutation at the age of 5 years. This patient later developed nontuberculous mycobacterial infection in bilateral renal cysts. No deaths related to BCG osteitis or its treatment were identified.

\section{DISCUSSION}

This study reports 21 children with laboratory-confirmed BCG osteitis treated at a single referral center in Korea from January 2007 to March 2018. The median age of symptom onset was 14.3 months. The most frequently administered BCG vaccine was the Tokyo-172 strain inoculated percutaneously $(76.2 \%)$, followed by the same strain administered intradermally $(19.0 \%)$. Only one child received the Danish strain by intradermal method. The most common symptoms were swelling, refusal to move the affected site, and pain, while fever was only accompanied in few. All of the patients received antimycobacterial medications for at least 12 months and all but one of the patients had surgical procedures either for diagnostic or therapeutic purposes. After treatment, most of the patients improved without sequelae.

The clinical manifestations of BCG osteitis in this study were comparable with those published in previous literatures. ${ }^{4,11}$ These clinical findings and the solitary well-demarcated osteolytic lesion frequently observed on the plain radiographs mimic acute bacterial osteomyelitis. ${ }^{12}$ As BCG osteitis is uncommon to easily come across clinicians' minds, it is important to recognize the distinctive features between bacterial osteomyelitis and BCG osteitis. Children with bacterial osteomyelitis commonly present with high fever and bone pain of abrupt onset. ${ }^{13}$ In this study, we found that BCG osteitis accompanied fever in $19 \%$ only and were diagnosed after a median of 29 days from symptom onset, and even 
longer delay in diagnosis of several months has been previously observed, underscoring the importance of clinical suspicion. Other important differential point is the affected age. Acute bacterial osteomyelitis can develop at any ages in children while BCG osteitis usually develops between 6 months and 5 years of age.

BCG osteitis is known to have a good prognosis and the overall outcome in this study was also favorable. ${ }^{1}$ During the median 3.2 years of follow-up, only one patient developed orthopedic complication related to BCG osteitis. One retrospective study conducted in Finland observed some sequelae in only 3\% of the BCG osteitis patients and a systemic review reported that $2.4 \%$ of children with BCG osteitis experience sequelae. ${ }^{11}$ However, more than one surgical interventions may be needed when the diagnosis is delayed due to persistent swelling and local spread of the infection as shown in this study, and patients receiving surgical interventions as part of the treatment seem to develop more complications than those receiving diagnostic procedures only. ${ }^{11}$ Moreover, patients with confirmed or suspected immunodeficiency could be prone to subsequent atypical infections in the previous BCG osteitis site and also present with multiple lesions. ${ }^{13} \mathrm{~A}$ recent study evaluating 160 former Finnish patients diagnosed 19-47 years ago in infancy reported a higher rate of $13.8 \%$ to have orthopedic complications, such as leg length discrepancy and chronic pain in the affected limb. ${ }^{14}$ Therefore, surveillance for serious adverse reactions following BCG vaccination and monitoring their long-term outcomes would be necessary.

In this study, it is important to note that $95.2 \%$ patients with BCG osteitis were vaccinated with the Tokyo-172 strain and $80.0 \%$ of them received the vaccine percutaneously via multiple puncture technique. Adverse reactions after BCG vaccination are known to depend on various factors including the vaccine strain, route of vaccine administration, and number of viable bacilli in the batch. ${ }^{1}$ BCG osteitis was widely reported in the 1970s primarily in Northern Europe where the occurrence of the disease was associated with the changes in the vaccine strain or the manufacturing method. The incidence of BCG osteitis in Sweden and Finland increased rapidly shortly after the manufacturer was changed from the Swedish Laboratory to the Statens Serum Institute, Copenhagen.15,16 Although the same Gothenburg strain was used, the incidence in Sweden and Finland increased from 2.5 to 33.0 per 100,000 and from 13.0 to 72.9 per 100,000 BCG-vaccinated infants which later decreased rapidly after the vaccine was replaced by the Copenhagen 1331 strain and the Glaxo-Evans strain, respectively. ${ }^{17}$ Interestingly, while the incidence of BCG osteitis following the Glaxo BCG vaccination was 6.4 per 100,000 from 1978 to 1988 in Finland, no cases were reported in the UK despite the use of the same vaccine. The underlying reason for the variation in incidence among different countries using the same BCG strain remains unclear.

The BCG Tokyo-172 strain is known to be less reactogenic than other strains and the incidence of BCG osteitis in Japan, where it is administered by percutaneous multiple puncture method, was reported as only 0.01 case per million in the 1980s, the lowest compared to other countries. ${ }^{18}$ During 1951-2004, the incidence still remained as low as 0.1 per million. ${ }^{7}$ However, the incidence of BCG osteitis in Japan increased by more than 10 -fold during 1998-2007 to 2 per million. The annual incidence was 2.2 cases per year between 1998 and 2007, and it further increased to 4.14 cases per year between 2005 and 2011.3,19 In Japan, BCG vaccination is not recommended during the first 3 months of age due to serious adverse events in immunodeficient infants, and the recommended age for vaccination was changed from 3-6 months to 5-8 months in 2013.19,20 In Taiwan, where The Tokyo-172 strain is administered intradermally, the incidence of BCG osteitis is reported to be much higher, 
estimated to be 3.68 per million during 2002-2006. ${ }^{8}$ With improved laboratory facility to differentiate $M$. bovis BCG from other $M$. tuberculosis complex species as well as enhanced surveillance, the incidence rapidly increased to 12.9 cases per million during 2005-2007 and then to 30.1 cases per million during 2008-2012.4,21 BCG vaccine was previously recommended for infants reaching 5 months of age, but the Taiwan Centers for Disease Control recently revised the schedule for infants aged 5 through 8 months to lower adverse event cases following BCG immunization. ${ }^{22}$

Based on this study of 21 children with BCG osteitis, the estimated incidence of BCG osteitis in Korea is also high. The Korean NIP recommends BCG vaccination for all neonates within 4 weeks after birth and about 96\%-97\% neonates are vaccinated. ${ }^{23}$ Considering the total number of about 4,900,000 births from 2007 to 2017 and that at least $95 \%$ of the newborns are vaccinated with BCG, the incidence of BCG osteitis during 2007-2017 in Korea would be estimated to be at least 4.08 cases per million vaccinations. ${ }^{24}$ Although the Korean NIP for BCG vaccination is based on the intradermal Danish strain, the proportion of percutaneous Tokyo-172 BCG vaccination is estimated to be $68 \%$ during recent 10 years, which is more than twice that of intradermal Danish BCG vaccination. ${ }^{25-27}$ The incidence of BCG osteitis following the percutaneous BCG Tokyo vaccine in Korea would be at least 4.44 cases per million and the incidence following the intradermal BCG Tokyo vaccine would be at least 8.27 per million. The data in this study and in previous studies conducted in Taiwan and Thailand imply that this BCG Tokyo-172 vaccine might be more virulent than expected, causing invasive disease in young immunocompetent children. ${ }^{5,28}$

Despite the high number of BCG osteitis observed in this single institute from 2007 to 2017, only 5 cases of BCG-related osteitis were actually reported and reimbursed through the government-operated vaccine injury compensation system, Korea National Vaccine Injury Compensation Program (KVICP), between 1995 and 2016.29-31 Even the five cases reported through the program do not match with our five patients who received intradermal BCG through the NIP. One main reason for the discordance would be that the percutaneous BCG Tokyo-172 vaccine, the most common vaccine used in Korea, was not included in the NIP during most of the study period, hence adverse reactions related to this vaccine strain was not monitored through the program. Hence, the overall serious adverse events related to BCG vaccination must have been underreported and its incidence underestimated. A comprehensive national surveillance system is needed to accurately monitor serious adverse reactions following both intradermal and percutaneous BCG vaccination to assess their safety in Korea.

This study has several limitations. This study was conducted in a single institute, retrospectively. However, as $90 \%$ of the patients were referred from all across the country, the data might have reflected the overall characteristics of BCG osteitis in Korea. Additionally, many children might not have been diagnosed due to lack of suspicion by clinicians and lack of conducting molecular testing even when suspected. Despite this concern of underdiagnosis, the estimated incidence of at least 4.08 per million based on a single center data itself depicts the numerous occurrences of BCG osteitis cases in Korea. The relative risk of BCG osteitis associated with the Tokyo-172 strain to the intradermal Danish strain could not be assessed in this study as the exact numbers of each vaccine strain used during the study years are unknown.

In conclusion, highly suspecting BCG osteitis based on clinical features is crucial in diagnosing and treating the disease promptly. So far, the incidence of BCG osteitis as well 
as other serious adverse reactions associated with BCG vaccination in Korea is likely to be underestimated due to limited national surveillance system. Active and thorough monitoring of vaccine safety is urgently required and the adverse reaction profiles should be further reflected in establishing safe and effective BCG vaccination policy in Korea.

\section{REFERENCES}

1. World Health Organization. BCG vaccines: WHO position paper - February 2018. Wkly Epidemiol Rec 2018;93(8):73-96. PUBMED

2. World Health Organization. BCG vaccine. WHO position paper. Wkly Epidemiol Rec 2004;79(4):27-38. PUBMED

3. Koyama A, Toida I, Nakata S. Osteitis as a complication of BCG vaccination. Kekkaku 2009;84(3):125-32. PUBMED

4. Chiu NC, Lin MC, Lin WL, Wang SY, Chi H, Huang LM, et al. Mycobacterium bovis BCG-associated osteomyelitis/osteitis, Taiwan. Emerg Infect Dis 2015;21(3):539-40. PUBMED | CROSSREF

5. Rermruay R, Rungmaitree S, Chatpornvorarux S, Brukesawan C, Wittawatmongkol O, Lapphra K, et al. Clinical features and outcomes of Bacille Calmette-Guérin (BCG)-induced diseases following neonatal BCG Tokyo-172 strain immunization. Vaccine 2018;36(28):4046-53. PUBMED | CROSSREF

6. Yamamoto S, Yamamoto T. Historical review of BCG vaccine in Japan. Jpn J Infect Dis 2007;60(6):331-6. PUBMED

7. Toida I, Nakata S. Severe adverse reactions after vaccination with Japanese BCG vaccine: a review. Kekkaku 2007;82(11):809-24.

PUBMED

8. Shue GC, Yang SL, Lee CD, Liu DP. Adverse events induced by BCG immunization in Taiwan. Taiwan Epidemiol Bull 2008;24(5):357-71.

9. Kim SH, Kim SY, Eun BW, Yoo WJ, Park KU, Choi EH, et al. BCG osteomyelitis caused by the BCG Tokyo strain and confirmed by molecular method. Vaccine 2008;26(34):4379-81. PUBMED | CROSSREF

10. Yoo WJ, Choi IH, Yun YH, Cho TJ, Cheon JE, Song MH, et al. Primary epiphyseal osteomyelitis caused by mycobacterium species in otherwise healthy toddlers. J Bone Joint Surg Am 2014;96(17):e145. PUBMED | CROSSREF

11. Lin WL, Chiu NC, Lee PH, Huang AS, Huang FY, Chi H, et al. Management of Bacillus Calmette-Guérin osteomyelitis/osteitis in immunocompetent children-a systematic review. Vaccine 2015;33(36):4391-7. PUBMED | CROSSREF

12. Peltola H, Pääkkönen M. Acute osteomyelitis in children. N Engl J Med 2014;370(4):352-60. PUBMED | CROSSREF

13. Goergens ED, McEvoy A, Watson M, Barrett IR. Acute osteomyelitis and septic arthritis in children.J Paediatr Child Health 2005;41(1-2):59-62. PUBMED | CROSSREF

14. Pöyhönen L, Pauniaho SL, Kröger L, Korppi M. Orthopedic complications in former Bacillus CalmetteGuérin osteitis patients. Pediatr Infect Dis J 2016;35(5):579-80. PUBMED | CROSSREF

15. Böttiger M, Romanus V, de Verdier C, Boman G. Osteitis and other complications caused by generalized BCG-itis. Experiences in Sweden. Acta Paediatr Scand 1982;71(3):471-8. PUBMED | CROSSREF

16. Lotte A, Wasz-Höckert O, Poisson N, Dumitrescu N, Verron M, Couvet E. BCG complications. Estimates of the risks among vaccinated subjects and statistical analysis of their main characteristics. Adv Tuberc Res 1984;21:107-93. PUBMED

17. Kröger L, Brander E, Korppi M, Wasz-Höckert O, Backman A, Kröger H, et al. Osteitis after newborn vaccination with three different Bacillus Calmette-Guérin vaccines: twenty-nine years of experience. Pediatr Infect Dis J1994;13(2):113-5.

PUBMED | CROSSREF 
18. Lotte A, Wasz-Höckert O, Poisson N, Dumitrescu N, Verron M, Couvet E. A bibliography of the complications of BCG vaccination. A comprehensive list of the world literature since the introduction of BCG up to July 1982, supplemented by over 100 personal communications. Adv Tuberc Res 1984;21:194-245. PUBMED

19. Ministry of Health, Labour and Welfare (JP). Q \& A on tuberculosis and BCG vaccine. http://www.mhlw. go.jp/seisakunitsuite/bunya/kenkou_iryou/kenkou/kekkaku-kansenshou/bcg/. Accessed June 18, 2018.

20. Ministry of Health, Labour and Welfare (JP). Review about the time of inoculation of BCG. http://www. mhlw.go.jp/stf/shingi/2r9852000002ones-att/2r9852000002onjx.pdf. Accessed June 18, 2018.

21. Jou R, Huang WL, Su WJ. Tokyo-172 BCG vaccination complications, Taiwan. Emerg Infect Dis 2009;15(9):1525-6. PUBMED | CROSSREF

22. Taiwan Centers for Disease Control. Beginning January 1, 2016, revised routine childhood immunization schedule to designate Bacille Calmette-Guérin (BCG) vaccine for infants reaching 5 months of age and recommend BCG vaccine for infants at 5-8 months of age. https://www.cdc.gov.tw/english/info. aspx?treeid=bc2d4e89b154059b\&nowtreeid=ee0a2987cfba3222\&tid=D396E3A542ECE908. Accessed June 18, 2018.

23. Korea Centers for Disease Control and Prevention. National immunization rate. http://www.cdc.go.kr/CDC/ info/CdcKrinfo0726.jsp?menuIds=HOME001-MNU1132-MNU2430-MNU2689. Accessed June 16, 2018.

24. Korean Statistical Information Service. Statistics by subject. http://kosis.kr/statisticsList/statisticsListIndex. do?menuId=M_01_01\&vwcd=MT_ZTITLE\&parmTabId=M_01_01. Accessed June 16, 2018.

25. Lee H, Dockrell HM, Kim DR, Floyd S, Oh SY, Lee JB, et al. The current status of BCG vaccination in young children in South Korea. Tuberc Respir Dis (Seoul) 2012;72(4):374-80. PUBMED | CROSSREF

26. Ministry of Health and Welfare (KR). Ministry of Health and Welfare Statistical Yearbook 2012. Seoul: Ministry of Health and Welfare; 2012.

27. Ministry of Health and Welfare (KR). Ministry of Health and Welfare Statistical Yearbook 2017. Seoul: Ministry of Health and Welfare; 2017.

28. Thamthitiwat S, Marin N, Baggett HC, Peruski LF, Kiatkulwiwat W, Panumatrasmee V, et al. Mycobacterium bovis (Bacille Calmette-Guérin) bacteremia in immunocompetent neonates following vaccination. Vaccine 2011;29(9):1727-30. PUBMED | CROSSREF

29. Lee HJ, Sohn YM, Kim JS, Kim YT, Lee JG, Choi BY. Analysis of the adverse events following immunization of the national compensation program and the surveillance system in Korea, 1995-2000. Korean J Pediatr Infect Dis 2001;8(2):135-49. CROSSREF

30. Kim MK, Lee YK, Kim TE, Kong I, Yang HJ, Suh ES. Surveillance and compensation claims for adverse events following immunization from 2011 to 2016 in the Republic of Korea. Clin Exp Vaccine Res 2017;6(2):146-55. PUBMED | CROSSREF

31. Korea Centers for Disease Control and Prevention. Guidelines for the Adverse Events Following Immunization Management. Cheongju: Korea Centers for Disease Control and Prevention; 2014. 